\title{
Preliminary Outcomes Following Application Of The Real-Time Navigation System Combined With Intraoperative Three-Dimensional C-Arm Computed Tomography For Zygomatico-Orbital Fracture Reconstruction
}

\author{
Yu-Ying Chu \\ Linkou Chang Gung Memorial Hospital \\ Jia-Ruei Yang \\ Linkou Chang Gung Memorial Hospital \\ Han Tsung Liao ( $\sim$ lia01211@gmail.com ) \\ Linkou Chang Gung Memorial Hospital \\ Bo-Ru Lai \\ Linkou Chang Gung Memorial Hospital
}

\section{Research Article}

Keywords: Computer-assisted navigation system, 3D C-arm, intraoperative computed tomography, zygomatico-orbital fracture, enophthalmos, facial asymmetry

Posted Date: August 24th, 2021

DOI: https://doi.org/10.21203/rs.3.rs-798592/v1

License: (c) (1) This work is licensed under a Creative Commons Attribution 4.0 International License. Read Full License 


\section{Preliminary Outcomes Following Application Of The Real-Time Navigation}

System Combined With Intraoperative Three-dimensional C-arm

Computed Tomography For Zygomatico-Orbital Fracture Reconstruction

Yu-Ying Chu ${ }^{1,2,3}$, Jia-Ruei Yang ${ }^{1,2,3}$, Bo-Ru Lai ${ }^{1,2,3}$, Han-Tsung Liao ${ }^{1,2,3,4^{*}}$

${ }^{1}$ Division of Trauma Plastic Surgery, Department of Plastic and Reconstructive Surgery, Chang Gung

Memorial Hospital, Taoyuan, 333, Taiwan

${ }^{2}$ Craniofacial Research Center, Chang Gung Memorial Hospital, Taoyuan, 333, Taiwan

${ }^{3}$ College of Medicine, Chang Gung University, Taoyuan, 333, Taiwan

${ }^{4}$ Department of plastic surgery, Xiamen Chang gung Hospital, Xiamen, 361000, China

*Corresponding author:

Han-Tsung Liao, MD, PhD., ORCID. lia01211@gmail.com

Professor

Division of Trauma Plastic Surgery, Department of Plastic and Reconstructive Surgery, Craniofacial

Research Center, Chang Gung Memorial Hospital, College of Medicine, Chang Gung University, 5

Fuxing Street, Taoyuan 333, Taiwan

Telephone: 886-3-328-1200 ext. 2946

Fax: 886-3-328-9582

E-mail:lia01211@gmail.com 


\begin{abstract}
This study analyzed the outcomes of zygomatico-orbital fracture reconstruction using the real-time navigation system with intraoperative three-dimensional (3D) C-arm computed tomography (CT). Fifteen patients with zygomatico-orbital or isolated orbital/zygoma fractures were enrolled in this prospective cohort. For zygoma reduction, the displacement at five key sutures and the differences between preoperative and intraoperative CT images were compared. For orbital reconstruction, the bilateral orbital volume differences in the anterior, middle, and posterior angles over the medial transitional buttress were measured. Two patients required implant adjustment once after the intraoperative 3D C-arm assessment. On comparing the preoperative and postoperative findings for the zygoma, the average sum of displacement was 19.48 (range, 5.1-34.65) vs. $\pm 1.96(0-3.95) \mathrm{mm}$ $(P<0.001)$ and the deviation index was $13.56(10-24.35)$ vs. $2.44(0.6-4.85)(P<0.001)$. For the orbit, the mean preoperative to postoperative bilateral orbital volume difference was 3.93 (0.35$10.95)$ vs. $1.05(0.12-3.61) \mathrm{mm}^{3}(P<0.001)$. The mean difference in the bilateral angles at the transition buttress was significantly decreased postoperatively at the middle and posterior one-third. The surgical navigation system with the intraoperative 3D C-arm can effectively improve the accuracy of zygomatico-orbital fracture reconstruction and decrease implant adjustment times.
\end{abstract}

\title{
Keywords
}

Computer-assisted navigation system; 3D C-arm, intraoperative computed tomography, zygomaticoorbital fracture, enophthalmos, facial asymmetry 


\section{Introduction}

Zygomatico-orbital (ZMO) fractures refer to injuries of the zygoma and the surrounding bone, including the orbit ${ }^{1}$. They are the most common type of orbital fractures ${ }^{2}$. Without adequate reduction and reconstruction of ZMO fractures, complications such as malar flattening, facial asymmetry, sensory disturbance, enophthalmos, hypoglobus, and diplopia may occur ${ }^{3,4}$. Therefore, a computer-assisted surgical navigation system and intraoperative computer tomography (CT) have been frequently proposed in the literature for the management of facial fracture surgery ${ }^{5-17}$. The intraoperative navigation system provides accurate reduction of the fracture bone that matches the virtually simulated position that was planned preoperatively ${ }^{6}$. It helps in confirming the position of the orbital implant and reduction of the displaced zygomatic fracture, optimizing the functional and aesthetic outcomes of orbit fractures ${ }^{10,18}$, and achieving postoperative symmetrical facial profiles for patients with zygomaticomaxillary complex (ZMC) fractures ${ }^{19}$. Although it involves real-time guidance, the accuracy is based on point-to-point confirmation instead of a match to the threedimensional (3D) anatomical architecture of reduced bone. On the other hand, intraoperative 3D Carm CT has also been well applied in craniofacial reconstruction. It can evaluate the adequacy of ZMC fracture reduction ${ }^{20}$ and ensure the implant position in ZMO fractures or isolated orbital fractures via a match to 3D architecture ${ }^{21}$. However, the use of the 3D C-arm is difficult with realtime guidance, and the intraoperative revision rate can be higher than $63 \%{ }^{17}$. Although the radiation dose of the intraoperative 3D C-arm CT is lower than that of conventional CT, frequent adjustments put the patient at risk of higher radiation exposure.

To combine the advantages and eliminate the disadvantages of these two techniques, our study aimed to determine the surgical outcomes of patients with ZMO fractures or isolated zygoma/orbital fractures who underwent reconstruction under the guidance of a surgical navigation system combined with the intraoperative 3D C-arm. 


\section{Results}

\section{Demographics and symptoms}

A total of 15 patients (4 males, 11 females) were enrolled in this study. The mean age was $39.2 \pm$ 16.0 years. Seven patients had ZMO fractures, seven had pure orbital fracture, and one had an isolated zygoma fracture. The fractures occurred on the right side in eight patients and on the left in seven. Thirteen fractures resulted from traffic accidents, one from a fall, and one from a human altercation. Seven patients received early/primary reconstruction, three underwent late reconstruction, and five had a secondary revision. After zygoma reduction, the MatrixMIDFACE ${ }^{\mathrm{TM}}$ Plating System was used for fixation, and a preformed mesh, titanium mesh, and Medpor were used for reconstruction and to reinforce the support of the fractured orbital floor and the medial wall. Intraoperatively, two patients required one adjustment of the implant and fixation plate position after 3D C-arm assessment. On clinical presentation, five patients had preoperative diplopia, five had enophthalmos, two had hypoglobus, four had EOM limitation, and eight had facial asymmetry. One patient reported traumatic optic neuropathy that resulted in visual loss, causing difficulty in diplopia assessment. There were no postoperative cases of facial asymmetry. Diplopia persisted postoperatively in three of the five patients (one had enophthalmos, one had hypoglobus, and one had EOM limitation), but the situation improved from its preoperative status. The diplopia appeared postoperatively only when looking in a single direction instead of in multiple directions as observed preoperatively (Table 1 ).

\section{Zygoma measurements}

In 2D fashion, the mean displacement values at each zygomatic suture line preoperatively and postoperatively are shown in Table 2. The average sum of preoperative and postoperative displacement was 19.48 (range, 5.1-34.65) vs. $\pm 1.96(0-3.95) \mathrm{mm}(P<0.001)$. In 3D fashion, the mean value of preoperative and postoperative differences between the surgical plan and CT images was also calculated. The DI preoperatively and postoperatively was 13.56 (10-24.35) vs. 2.44 (0.64.85) $\mathrm{mm}(P<0.001)$ (Table 2).

\section{Orbit measurement}

The mean values of the preoperative and postoperative bilateral orbital volume difference were 3.93 $(0.35-10.95)$ vs. $1.05(0.12-3.61) \mathrm{mm}^{3}(P<0.001)$. The mean values of the bilateral angle difference at the transition zone of the orbital buttress preoperatively and postoperatively were 15.94 (0.1-89) vs. $5.51(0-30.1)(P=0.134)$ degrees at the anterior one-third, $35.94(0.5-144)$ vs. $5.74(0.3-21.4)(P$ $=0.016)$ degrees at the middle, and $41.18(5.1-127.3)$ vs. $5.15(0-18)(P=0.002)$ degrees at the posterior one-third, respectively (Table 3 ).

\section{Case presentation}

A 59-year-old female patient who met with a motorcycle accident presented with a right face 
contusion. Preoperative CT revealed a right zygoma tetrapod fracture with a concomitant right orbital floor blow-out fracture. Clinically, right malar depression with ecchymosis and mild enophthalmos with subconjunctival hemorrhage were documented. No EOM limitation, diplopia, or visual acuity deficiency was noted. The patient had also sustained a Colle's fracture of the right forearm, but she had no known systemic underlying disease.

The ideal preoperative plan was designed by the BrainLAB software. Semiautomated segmentation volumetric objects were created on the unaffected side (blue)(Fig.1), superimposed onto the preoperative CT images, and mirrored to the affected side (red). Under satisfactory general anesthesia, the head and neck area were prepared and draped in a sterile manner. Surface matching registration was conducted successfully by verification with laser pointer localization of the upper incisors. Then, right upper mucogingival and subciliary incisions were made, and the right zygomacomminuted fracture and right orbital floor blow-out fracture were located. The bone was reduced under real-time surgical navigation guidance and fixed with mini plates over the ZF junction, infraorbital rim, lateral ZM buttress, and anterior maxillary wall. The orbital floor blow-out fracture was re-established with titanium mesh under navigation guidance. The real-time position of the reduced zygoma prominence and orbital floor was confirmed by the laser pointer in the axial, coronal, and sagittal views. Thereafter, intraoperative 3D C-arm CT was used to verify the final position, with imaging of the entire facial skeleton. The CT images showed satisfactory conformity with the presurgical design. The wound was repaired in layers. At the 6-month follow-up, the patient revealed symmetric bilateral malar and eyeball projections. No postoperative complications, such as diplopia, EOM limitation, enophthalmos, or hypoglobus, were reported (Fig. 2). 


\section{Discussion}

Our study results revealed that the use of the computer-assisted surgical navigation system for presurgical planning and intraoperative real-time guidance with 3D C-arm CT provides accurate and effective zygoma reduction and orbital fracture reconstruction. Among the eight patients with a facial fracture involving the zygoma, the postoperative sum of displacement in each zygomatic suture decreased from 19.48 to $1.96 \mathrm{~mm}$ and the DI decreased from 13.56 to $2.44 \mathrm{~mm}$. The final distances between each zygomatic suture point or zygomatic prominence surface point and that of the presurgical plan was less than $2 \mathrm{~mm}$, which is clinically undetectable appearance-wise.

All the patients had obvious preoperative facial asymmetry with malar depression or zygomatic arch protrusion. After the operation, facial symmetry improved in all patients. For orbit assessment, the orbital volume difference between the affected and unaffected eyes decreased from 3.93 to 1.05 $\mathrm{mm}^{3}$ after the operation. An increase in orbital volume causes enophthalmos. It has been reported that enophthalmos of $<2 \mathrm{~mm}$ is not clinically recognized ${ }^{22-24}$. The correlation between the orbital volume difference amount and enophthalmos degree ranges from 0.47 to $0.87 \mathrm{~mm}$ per $1-\mathrm{mm}^{3}$ volume expansion ${ }^{25-27}$. Theoretically, the $1.05-\mathrm{mm}^{3}$ volume difference is less likely to result in clinically significant enophthalmos.

Five of the fourteen patients with fractures involving the orbit presented with preoperative enophthalmos. Only one patient, who received secondary reconstruction, had residual mild enophthalmos. This may be related to the cicatricial effect and fat atrophy after inadequate primary manipulation of the orbital fracture ${ }^{28}$. For the angles of the transition zone of the orbital buttress, the middle and posterior one-third showed significant bilateral angle differences preoperatively and postoperatively. However, the difference in the anterior one-third only showed a decreasing trend. This may result from the small sample size and/or the fact that fractures of the medial wall and floor mostly affect the middle and posterior regions; thus, the bilateral angle difference of the anterior one-third was relatively less than those the other two locations, causing insignificant changes after the operation.

Real-time surgical navigation systems have proved to be effective and beneficial in the clinical application of complex midfacial fracture reduction and complex orbital fracture reconstruction ${ }^{29}$. For reduction of the acute zygomatic fracture, under intraoperative navigation guidance, the deviation amount of each suture in the zygomatic fracture can be reduced to $<2 \mathrm{~mm}^{11,30}$. For orbital reconstruction, the rates of late and secondary surgery are more frequent than the rates for zygoma reconstruction, causing enophthalmos if left untreated. Correction is considered challenging if the fracture involves the transition zone of the orbital buttress. Previous studies have demonstrated the strong advantages of navigation guidance in improving precision and functional and aesthetic outcomes, facilitating intraoperative visualization, and avoiding vital structure injury in the reestablishment of extensive orbital fractures ${ }^{10,31,32}$. He et al. also reported that navigation-guided surgery with a 3D model and titanium mesh with Medpor implants are optimal for treating late ZMO fractures with severe enophthalmos ${ }^{33}$. In a recent study assessing extensive orbital reconstruction 
in a cadaveric model, real-time navigation was the fastest, most accurate, and safe method for orbital implant positioning ${ }^{34}$. Although navigation can be used to verify the position of implants or reduction in real-time, it cannot provide the most updated reconstructed bone information if the anatomy is changed during the surgery ${ }^{35}$. Therefore, the concomitant application of intraoperative 3D C-arm CT compensates for the inadequacy of the navigation system.

Intraoperative CT is also an effective tool for improving surgical outcomes of zygoma or orbital fractures. The important benefit of its usage is the integration of a wide flat-panel detector, which facilitates visualization of the entire anatomical craniofacial structure ${ }^{14}$. During the operation, it plays an important role in assessing the precision and accuracy of reduction, confirming the implant position in the orbit or the protrusion of the zygoma, and allowing immediate adjustment. In addition, the radiation exposure for midfacial imaging was much higher in the conventional multislice CT modality than in cone-beam CT systems ${ }^{36}$. Even for high-contrast structures, cone-beam CT could lessen the radiation exposure by about $50 \%$ of that used by conventional CT following particular parameters ${ }^{14}$. Using large-area flat-panel detectors, a detailed high-resolution 3D image of the craniofacial structure can be obtained ${ }^{37}$.

Using an intraoperative 3D C-arm system, Wilde et al. found that 4 of 21 patients with ZMO complex fractures had inadequate repair or reduction that required adjustment ${ }^{38}$. Chen et al. reported that 7 of 11 patients who underwent secondary or delayed primary orbital reconstruction with the aid of intraoperative CT required intraoperative adjustment ${ }^{32}$. Nonetheless, the avoidance of radiation exposure from postoperative imaging, surgical morbidity ${ }^{38}$, and another revision surgery exceeds the disadvantage of an increased operation time ${ }^{17,21}$.

Although intraoperative 3D C-arm CT ameliorates the surgical results in ZMO fractures ${ }^{21}$, it does not involve a real-time guidance because each implant position correction requires repeated intraoperative imaging, which take time to process. To sustain the advantages and eliminate the disadvantages of the surgical navigation system and intraoperative 3D C-arm CT, these two techniques were coupled for midface fracture reconstruction. In 2011, Scolozzi et al. reported the combination of "mirroring" computational presurgical planning, intraoperative surgical navigation system, and C-arm cone-beam CT with a flat-panel detector for management of primary and secondary midfacial fractures in five patients ${ }^{39}$. For further proof of the principle, our study quantitatively measured the surgical outcomes of parameters in the zygoma and the orbit, reflecting the standard usage of the navigation system as a real-time guide and the 3D C-arm CT as the final verification of the reconstruction results. With intraoperative $\mathrm{CT}$ alone, the correction rate during surgery can be as high as $63.3 \%{ }^{17}$. When coupled with the navigation system and 3D C-arm CT, the intraoperative correction rate was $13.3 \%$, which is much lower compared with that observed for CT alone during surgery.

In conclusion, the surgical navigation system combined with intraoperative 3D C-arm CT for the management of ZMO fractures has the advantages of both the techniques, thereby facilitating anatomical landmark reduction and functional reconstruction effectively, improving the accuracy and precision of surgery, and decreasing the rate of implant position adjustment. 


\section{Methods}

\section{Patients}

Fifteen patients ( 4 males and 11 females) with ZMO fractures or isolated orbital (floor and medial wall)/zygoma fractures were recruited between September 2020 and June 2021. Patients aged below 18 years or who presented with other facial fractures were excluded. All the patients underwent ZMO reconstruction under the guidance of a surgical navigation system combined with intraoperative 3D C-arm (Ziehm Vision RFD 3D) (Ziehm Imaging GmbH, Nuremberg, Germany). When a patient's first reconstruction was performed within 30 days of injury, it was defined as early or primary reconstruction, and reconstruction performed more than 30 days after the injury was defined as late or delayed primary reconstruction. Secondary surgery was defined when the patient had received previous reduction or reconstruction. Preoperative and postoperative signs and symptoms, including enophthalmos, hypoglobus, facial asymmetry, extraocular muscle movement (EOM) limitation, and diplopia were documented. This prospective study was approved by the Institutional Review Board of Chang Gung Memorial Hospital, Linkou Branch, Taiwan (IRB: 202000509A3C501). The procedures used in this study adhere to the tenets of the Declaration of Helsinki. Informed consent was obtained from all patients.

\section{Materials}

We obtained preoperative computed tomography (CT) images of the facial bone on the day of surgery. The collimation/slice thickness was $1 \mathrm{~mm}$. Digital Imaging and Communications in Medicine data was exported for further virtual planning and simulation in the 3D modeling software (Brain LAB AG, Munich, Germany). After loading the image data, the alignment of the cranium was adjusted according to the Frankfort Horizontal plane (FH) and vertical midline. The subsequential objects of the unaffected orbit and zygoma were created by semiautomated segmentation. The digital data was superimposed to the bone of the unaffected side and then mirrored to the affected side for surgical planning. We used the BrainLAB Vector Vision navigation platform as a guide to confirm the adequacy of zygomatic reduction or orbit reconstruction during operation. A tool adapter as skull reference was fixed to the parietal bone of the unaffected side. Three reflective plastic spheres with glass-grain coating (Latero Reference Star, BrainLAB) were secured to the adapter. A laser pointer (Ztouch Brain $L A B$ ) was used to surf and scan the frontal, periorbital, and nasal skin randomly for surface matching. When verifying the registration accuracy between CT images and the patient's anatomical structure, we placed the navigation pointer tip on the surface of the upper central incisors as a landmark to check the precision of the anatomical position in three dimensions (Fig. 3). A difference of $<1 \mathrm{~mm}$ between the preoperative imaging and the landmarks of the navigation pointer was considered acceptable.

\section{Surgical procedure and intraoperative 3D C-arm}

With the patient in the supine position under general anesthesia, the head and neck area were 
sterilized with a povidone-iodine solution, and the oral cavity was prepared with antiseptic gargles. According to the fracture patterns, incisions were made on the mucosa of the upper gingivobuccal sulcus for zygoma reduction or on the inferior conjunctiva or subciliary area for orbital reconstruction. To ensure precise reduction, the surgical navigation system was used to examine every suture line or critical point repeatedly until it matched the preoperative plan (Fig. 1). The MatrixMIDFACE ${ }^{\mathrm{TM}}$ Plating System was used for fixation of the fractured zygoma after reduction. A preformed mesh, titanium mesh, and Medpor were used for reconstruction of the fractured orbital floor and medial wall. In some patients, when the mesh alone was unable to ideally match the presurgical plan due to poor support by the surrounding bone, additional Medpor pieces (Porex Surgical Inc, Atlanta, Ga) were positioned under the mesh at the orbital buttress of the transition zone or at posterior ledge of the orbital floor.

After plating, intraoperative 3D C-arm CT was performed to determine the adequacy of zygoma reduction and orbital reconstruction. For assessment of the ZMO bone position, the flap panel detector 3D (low dose modifier) was used. An identical field of view was chosen to cover the entire skull bone. The uniform tube voltage of the 3D scan was set at $80 \mathrm{kV}$. The tube current [mA] was automatically dependent on the X-rayed object. The 3D scan images were acquired via a single rotation of the $\mathrm{C}$-arm for $180^{\circ}$ to obtain a complete dataset. The acquired images were immediately merged with the presurgical plan by the BrainLAB software to compare the conformity of the intraoperative reconstruction result and the plan (Fig. 4). Adjustments were then made as needed.

\section{Zygoma measurement}

Using the facial bone CT image, the preoperative and intraoperative surgical landmarks were evaluated based on the degree of displacement at the following five key suture lines in the 2D fashion: the zygomaticofrontal (ZF), inferior orbital rim (IO), zygomaticosphenoidal (ZS), zygomaticomaxillary $(\mathrm{ZM})$, and zygomaticotemporal $(\mathrm{ZT})^{40}$. The bone displacement value of each suture line was summed for comparison of preoperative and postoperative findings. In the 3D fashion, a CT scan in the axial view parallel to the FH plane that displayed the entire zygoma shape was chosen for evaluation. The presurgical plan was displayed simultaneously. We identified the infraorbital foramen and the base of the zygomatic arch upon CT. Between these two landmarks, the five points $(Z 1, Z 2, Z 3, Z 4$, and $Z 5)$, which were evenly distributed based on the angle of degree, were defined on the surface of zygoma. We also defined the point $(0)$ as the intersection of the vertical midline and the horizontal line that passed through the bilateral zygoma arch base. The Z1O line that intersected at the surface of the zygoma object in presurgical planning was defined as $\mathrm{P} 1$. The other points, i.e., P2 to P5, were defined in the same manner. We measured the preoperative and postoperative distances between $\mathrm{Z} 1$ and $\mathrm{P} 1, \mathrm{Z} 2$ and $\mathrm{P} 2, \mathrm{Z3}$ and $\mathrm{P} 3, \mathrm{Z4}$ and $\mathrm{P} 4$, and $\mathrm{Z} 5$ and $\mathrm{P} 5$ on the same cut of axial view and defined the summation of these five distances as the "deviation index"

(DI) to indicate the difference between the actual zygoma position and the ideal surgical plan (Fig. 5). 


\section{Orbit measurements}

For the patient with fractures that involved the orbital floor and medial wall, the orbital volume and bilateral angles of the orbital buttress of transition zone at the anterior, middle, and posterior onethird were measured preoperatively and intraoperatively. The images were also assessed by the BrainLAB software. The head was aligned, as previously described. The objects of the bilateral orbital cavity were created by autosegmentation. Fine adjustment of the object's borders in the coronal, axial, and sagittal views was performed via an inbuilt function for optimizing the conformity to the bone margins. After shaping, the orbital volume of both the eyes was obtained (Fig. 6). For angle assessment of the transition zone of the orbital buttress, three points were identified on the orbital floor at the sagittal view. These three points equally divided the floor length and defined the plane of the anterior one-third, posterior one-third, and middle in the coronal view. The angles of the transition zone of the medial orbital buttress were measured from the coronal view and compared bilaterally (Fig. 7).

\section{Statistical analysis}

Demographic data are described as number or mean (range, min-max). Preoperative and postoperative data were compared using the Wilcoxon signed-rank test. The results represented statistical significance at a $P$ value of $<0.05$. 


\section{References}

1.Ellis, E., 3rd \& Perez, D. An algorithm for the treatment of isolated zygomatico-orbital fractures. J Oral Maxillofac Surg. 72, 1975-1983, doi:10.1016/j.joms.2014.04.015 (2014).

2.Ellis, E., 3rd, el-Attar, A. \& Moos, K. F. An analysis of 2,067 cases of zygomatico-orbital fracture. J Oral Maxillofac Surg. 43, 417-428, doi:10.1016/s0278-2391(85)80049-5 (1985).

3.Kristensen, S. \& Tveterås, K. Zygomatic fractures: classification and complications. Clin Otolaryngol Allied Sci. 11, 123-129, doi:10.1111/j.1365-2273.1986.tb00117.x (1986).

4.Balle, V., Christensen, P. H., Greisen, O. \& Jørgensen, P. S. Treatment of zygomatic fractures: a follow-up study of 105 patients. Clin Otolaryngol Allied Sci. 7, 411-416, doi:10.1111/j.13652273.1982.tb01405.x (1982).

5.Schmelzeisen, R. et al. Navigation-aided reconstruction of medial orbital wall and floor contour in cranio-maxillofacial reconstruction. Injury. 35, 955-962, doi:10.1016/j.injury.2004.06.005 (2004).

6.Westendorff, C., Gülicher, D., Dammann, F., Reinert, S. \& Hoffmann, J. Computer-assisted surgical treatment of orbitozygomatic fractures. J Craniofac Surg. 17, 837-842, doi:10.1097/01.scs.0000221523.80292.93 (2006).

7.Pham, A. M., Rafii, A. A., Metzger, M. C., Jamali, A. \& Strong, E. B. Computer modeling and intraoperative navigation in maxillofacial surgery. Otolaryngol Head Neck Surg. 137, 624-631, doi:10.1016/j.otohns.2007.06.719 (2007).

8.Schramm, A. et al. Computer-assisted therapy in orbital and mid-facial reconstructions. Int J Med Robot. 5, 111-124, doi:10.1002/rcs.245 (2009).

9.Yu, H., Shen, G., Wang, X. \& Zhang, S. Navigation-guided reduction and orbital floor reconstruction in the treatment of zygomatic-orbital-maxillary complex fractures. J Oral Maxillofac Surg. 68, 28-34, doi:10.1016/j.joms.2009.07.058 (2010).

10.Yang, J. R. \& Liao, H. T. Functional and Aesthetic Outcome of Extensive Orbital Floor and Medial Wall Fracture via Navigation and Endoscope-assisted Reconstruction. Ann Plast Surg. 82, S77-s85, doi:10.1097/sap.0000000000001700 (2019).

11.Yang, C., Lee, M. C., Pan, C. H., Chen, C. H. \& Chen, C. T. Application of Computer-Assisted Navigation System in Acute Zygomatic Fractures. Ann Plast Surg. 82, S53-s58, doi:10.1097/sap.0000000000001721 (2019).

12. Hoelzle, F. et al. Intraoperative computed tomography with the mobile CT Tomoscan M during surgical treatment of orbital fractures. Int J Oral Maxillofac Surg. 30, 26-31, doi:10.1054/ijom.2000.0014 (2001).

13. Heiland, M., Schulze, D., Blake, F. \& Schmelzle, R. Intraoperative imaging of zygomaticomaxillary complex fractures using a 3D C-arm system. Int J Oral Maxillofac Surg. 34, 369-375, doi:10.1016/j.ijom.2004.09.010 (2005).

14.Pohlenz, P. et al. Intraoperative cone-beam computed tomography in oral and maxillofacial surgery using a C-arm prototype: first clinical experiences after treatment of zygomaticomaxillary complex fractures. J Oral Maxillofac Surg. 67, 515-521, doi:10.1016/j.joms.2008.06.086 (2009). 
15.Rabie, A., Ibrahim, A. M., Lee, B. T. \& Lin, S. J. Use of intraoperative computed tomography in complex facial fracture reduction and fixation. J Craniofac Surg. 22, 1466-1467, doi:10.1097/SCS.0b013e31821d1982 (2011).

16.Cuddy, K. et al. Management of Zygomaticomaxillary Complex Fractures Utilizing Intraoperative 3-Dimensional Imaging: The ZYGOMAS Protocol. J Oral Maxillofac Surg. 79, 177-182, doi:10.1016/j.joms.2020.08.028 (2021).

17.Shyu, V. B., Chen, H. H., Chen, C. H. \& Chen, C. T. Clinical outcome following intraoperative computed tomography-assisted secondary orbital reconstruction. J Plast Reconstr Aesthet Surg. 74, 341-349, doi:10.1016/j.bjps.2020.08.049 (2021).

18.Shin, H. S., Kim, S. Y., Cha, H. G., Han, B. L. \& Nam, S. M. Real Time Navigation-Assisted Orbital Wall Reconstruction in Blowout Fractures. J Craniofac Surg. 27, 370-373, doi:10.1097/scs.0000000000002410 (2016).

19.Bao, T. et al. Quantitative assessment of symmetry recovery in navigation-assisted surgical reduction of zygomaticomaxillary complex fractures. J Craniomaxillofac Surg. 47, 311-319, doi:10.1016/j.jcms.2018.12.003 (2019).

20.Singh, M., Ricci, J. A. \& Caterson, E. J. Use of Intraoperative Computed Tomography for Revisional Procedures in Patients with Complex Maxillofacial Trauma. Plast Reconstr Surg Glob Open. 3, e463, doi:10.1097/gox.0000000000000455 (2015).

21.Sharma, P., Rattan, V., Rai, S. \& Chhabbra, R. Does Intraoperative Computed Tomography Improve the Outcome in Zygomatico-Orbital Complex Fracture Reduction? J Maxillofac Oral Surg. 20, 189-200, doi:10.1007/s12663-020-01420-0 (2021).

22.Koo, L., Hatton, M. P. \& Rubin, P. A. When is enophthalmos "significant"? Ophthalmic Plast Reconstr Surg. 22, 274-277, doi:10.1097/01.iop.0000226865.22184.6a (2006).

23.Gart, M. S. \& Gosain, A. K. Evidence-based medicine: Orbital floor fractures. Plast Reconstr Surg. 134, 1345-1355, doi:10.1097/prs.0000000000000719 (2014).

24.Sung, Y. S., Chung, C. M. \& Hong, I. P. The Correlation between the Degree of Enophthalmos and the Extent of Fracture in Medial Orbital Wall Fracture Left Untreated for Over Six Months: A Retrospective Analysis of 81 Cases at a Single Institution. Arch Plast Surg. 40, 335-340, doi:10.5999/aps.2013.40.4.335 (2013).

25.Whitehouse, R. W., Batterbury, M., Jackson, A. \& Noble, J. L. Prediction of enophthalmos by computed tomography after 'blow out' orbital fracture. Br J Ophthalmol. 78, 618-620, doi:10.1136/bjo.78.8.618 (1994).

26. Raskin, E. M. et al. Prediction of late enophthalmos by volumetric analysis of orbital fractures. Ophthalmic Plast Reconstr Surg. 14, 19-26, doi:10.1097/00002341-199801000-00006 (1998).

27.Ahn, H. B. et al. Prediction of enophthalmos by computer-based volume measurement of orbital fractures in a Korean population. Ophthalmic Plast Reconstr Surg. 24, 36-39, doi:10.1097/IOP.0b013e31815eb7ce (2008).

28.Strong, E. B. Orbital fractures: pathophysiology and implant materials for orbital reconstruction. Facial Plast Surg. 30, 509-517, doi:10.1055/s-0034-1394099 (2014). 
29.Sukegawa, S., Kanno, T. \& Furuki, Y. Application of computer-assisted navigation systems in oral and maxillofacial surgery. Jpn Dent Sci Rev. 54, 139-149, doi:10.1016/j.jdsr.2018.03.005 (2018).

30.Chu, Y.-Y., Yang, J.-R., Pek, C.-H. \& Liao, H.-T. Application of Real-Time Surgical Navigation for Zygomatic Fracture Reduction and Fixation. J Plast Reconstr Aesthet Surg, doi:10.1016/j.bjps.2021.05.052.

31.Tarsitano, A., Badiali, G., Pizzigallo, A. \& Marchetti, C. Orbital Reconstruction: Patient-Specific Orbital Floor Reconstruction Using a Mirroring Technique and a Customized Titanium Mesh. J Craniofac Surg. 27, 1822-1825, doi:10.1097/scs.0000000000002907 (2016).

32.Chen, C. T. et al. Clinical outcomes for minimally invasive primary and secondary orbital reconstruction using an advanced synergistic combination of navigation and endoscopy. J Plast Reconstr Aesthet Surg. 71, 90-100, doi:10.1016/j.bjps.2017.08.018 (2018).

33. He, D. et al. Orbitozygomatic fractures with enophthalmos: analysis of 64 cases treated late. $J$ Oral Maxillofac Surg. 70, 562-576, doi:10.1016/j.joms.2011.02.041 (2012).

34.Schreurs, R., Becking, A. G., Jansen, J. \& Dubois, L. Advanced Concepts of Orbital Reconstruction: A Unique Attempt to Scientifically Evaluate Individual Techniques in Reconstruction of Large Orbital Defects. Atlas Oral Maxillofac Surg Clin North Am. 29, 151-162, doi:10.1016/j.cxom.2020.10.003 (2021).

35.Hsu, L., Fried, M. P. \& Jolesz, F. A. MR-guided endoscopic sinus surgery. AJNR Am J Neuroradiol. 19, 1235-1240 (1998).

36.Schulze, D., Heiland, M., Thurmann, H. \& Adam, G. Radiation exposure during midfacial imaging using 4- and 16-slice computed tomography, cone beam computed tomography systems and conventional radiography. Dentomaxillofac Radiol. 33, 83-86, doi:10.1259/dmfr/28403350 (2004).

37.Schaaf, H. et al. High resolution imaging of craniofacial bone specimens by flat-panel volumetric computed tomography. J Craniomaxillofac Surg. 36, 234-238, doi:10.1016/j.jcms.2008.02.006 (2008).

38.Wilde, F. et al. Intraoperative imaging with a 3D C-arm system after zygomatico-orbital complex fracture reduction. J Oral Maxillofac Surg. 71, 894-910, doi:10.1016/j.joms.2012.10.031 (2013).

39.Scolozzi, P. \& Terzic, A. "Mirroring" computational planning, navigation guidance system, and intraoperative mobile C-arm cone-beam computed tomography with flat-panel detector: a new rationale in primary and secondary treatment of midfacial fractures? J Oral Maxillofac Surg. 69, 1697-1707, doi:10.1016/j.joms.2010.07.049 (2011).

40.Mahmoud, S. M., Liao, H. T. \& Chen, C. T. Aesthetic and Functional Outcome of Zygomatic Fractures Fixation Comparison With Resorbable Versus Titanium Plates. Ann Plast Surg. 76 Suppl 1, S85-90, doi:10.1097/sap.0000000000000700 (2016). 


\section{Acknowledgments}

We are grateful to the Chang Gung Memorial Hospital under Grant Agreement No. CMRPG3K1201.

\section{Authors' contributions statement}

All authors contributed to the study's conception and design. Material preparation, data collection, and analysis were performed by Y.Y.C., J.R.Y., B.R.L. and H.T.L. The first draft of the manuscript was written by Y.Y.C., and all authors commented on previous versions of the manuscript. All authors read and approved the final manuscript.

\section{Funding}

The research leading to these results received funding from Chang Gung Memorial Hospital under Grant Agreement No. CMRPG3K1201.

\section{Conflicts of interest/Competing interests}

The authors have no conflicts of interest to declare that are relevant to the content of this article.

\section{Availability of data and material}

All authors are sure that all data and materials support the published claims and comply with field standards.

\section{Code availability}

All authors are sure that the software application supports the published claims and comply with field standards.

\section{Ethics approval}

The study was approved by the Institutional Review Board of Chang Gung Memorial Hospital, Linkou Branch, Taiwan (IRB: 202000509A3C501). The procedures used in this study adhere to the tenets of the Declaration of Helsinki.

\section{Consent to participate}

Informed consent was obtained from all individual participants included in the study.

\section{Consent for publication}

Patients signed informed consent regarding publishing their data and photographs. 


\section{Figure legends}

Fig. 1 (a) Intraoperative real-time navigation for confirmation of zygomatic bone position at the zygomatic prominence

The semiautomated segmentation objects of the digital template were created on the unaffected side (blue) and superimposed onto the preoperative CT images, then they were mirrored to the affected side (red), resulting in an ideal presurgical plan with symmetrical positioning of the bones. The real-time position of the reduced zygoma prominence was confirmed by the laser pointer in the axial, coronal, and sagittal views.

$\mathrm{CT}$, computed tomography

(b) Intraoperative real-time navigation for confirmation of zygomatic bone position near the zygomaticofrontal junction

The real-time position of the reduced zygoma near the zygomaticofrontal junction was confirmed by the laser pointer in the axial, coronal, and sagittal views.

(c) Intraoperative real-time navigation for confirmation of reconstructed implant position on the orbital floor.

The real-time position of the reconstructed implant is shown, as verified on the orbital floor by the laser pointer in the axial, coronal, and sagittal views.

\section{Fig. 2 Clinical outcomes of facial symmetry and enophthalmos}

The patient presented with right malar depression and mild enophthalmos after zygomatico-orbital fractures in the preoperative frontal and axial views $(\mathbf{a}, \mathbf{b})$. Six months after surgery, the patient reported symmetric projection of both the eyeballs and bilateral malar eminence (c, d), and the results were esthetically satisfactory.

\section{Fig. 3 Presurgical verification of the accuracy of reduction}

The laser pointer tip was placed on the surface and tip of the upper central incisors as landmarks to determine the precision of the anatomical position in three dimensions. A difference of $<1 \mathrm{~mm}$ between the preoperative imaging and the landmarks of the navigation pointer was considered acceptable.

Fig. 4 Fusion of preoperative computed tomography image, navigation presurgical planning, and intraoperative 3D C-arm computed tomography image

The intraoperative 3D C-arm computed tomography image is merged partially to the objects of navigation planning at the unaffected side (left) on the coronal and axial view (a, b). The updated position of the reduced zygoma and reconstructed orbital floor with implants (c, d). From different panels of the sagittal view, the preoperative images $(\mathbf{e}, \mathbf{f})$ and intraoperative images after implant 
positioning $(\mathbf{g}, \mathbf{h})$. The bone and implant contour accurately matched the presurgical planning.

\section{Fig. 5 Measurements of zygoma conformity according to presurgical planning}

The infraorbital foramen (yellow asterisk) and base of zygomatic arch (red asterisk) were identified on $\mathrm{CT}$. The five points (Z1, Z2, Z3, Z4, and Z5) (violet points) on the zygomatic surface were defined and evenly distributed based on its angle of degree. The $O$ point (yellow point) is defined as the intersection of the vertical midline and the horizontal line that passed through the bilateral zygoma arch base. The $\mathrm{Z1O}$ line (yellow dotted line) that intersected at the surface of the zygoma object during presurgical planning is defined as P1 (green point). The other points P2 to P5 (green points) are defined in the same manner. The preoperative and postoperative distances to virtual planning images between $\mathrm{Z} 1$ and $\mathrm{P} 1, \mathrm{Z} 2$ and $\mathrm{P} 2, \mathrm{Z3}$ and $\mathrm{P} 3, \mathrm{Z} 4$ and $\mathrm{P} 4$, and $\mathrm{Z} 5$ and $\mathrm{P} 5$ were measured.

\section{Fig. 6 Measurements of bilateral orbital volume}

The orbital volume was measured by the built-in function of object auto segmentation of the bilateral orbital cavity in the BrainLAB software. The fine adjustment of the object border was performed manually, and the orbital volume was obtained.

\section{Fig. 7 Measurements of bilateral angles of the transitional zone of the orbital buttress}

The anterior, middle, and posterior points (red stars) are identified and evenly distributed on the orbital floor at the transitional buttress on the sagittal plane. The angles of the transitional buttress were measured on the coronal plane bilaterally. The figure shows that the bilateral angles of the posterior area are determined. 
Tables

Characteristics

No. of patients

\begin{tabular}{|c|c|}
\hline & n (\%) \\
\hline Age (y/o) (mean, (SD)) & $39.2(16.0)$ \\
\hline \multicolumn{2}{|l|}{ Sex } \\
\hline Male & $4(26.7)$ \\
\hline Female & $11(73.3)$ \\
\hline \multicolumn{2}{|l|}{ Trauma mechanism } \\
\hline Traffic accident & $13(86.7)$ \\
\hline Fall & $1(6.67)$ \\
\hline Human altercation & $1(6.67)$ \\
\hline \multicolumn{2}{|l|}{ Fracture site } \\
\hline Right & $8(53.3)$ \\
\hline Left & $7(46.7)$ \\
\hline \multicolumn{2}{|l|}{ Fracture pattern } \\
\hline Pure orbital & $7(46.7)$ \\
\hline Pure zygoma & $1(6.7)$ \\
\hline Zygomatico-orbital & $7(46.7)$ \\
\hline \multicolumn{2}{|l|}{ Surgical timing } \\
\hline Early & $7(46.7)$ \\
\hline Late & $3(20)$ \\
\hline Secondary & $5(33.3)$ \\
\hline \multicolumn{2}{|c|}{ Intraoperative adjustment } \\
\hline Yes & $2(13.3)$ \\
\hline No & $13(86.6)$ \\
\hline \multicolumn{2}{|l|}{ Preoperative symptoms } \\
\hline Facial asymmetry & $8(53.3)$ \\
\hline Diplopia & $5(33.3)$ \\
\hline EOM limitation & $4(26.7)$ \\
\hline Enophthalmos & $5(33.3)$ \\
\hline Hypoglobus & $2(13.3)$ \\
\hline \multicolumn{2}{|l|}{ Postoperative symptoms } \\
\hline Facial asymmetry & $0(0)$ \\
\hline Diplopia & $3(20)$ \\
\hline EOM limitation & $1(6.7)$ \\
\hline Enophthalmos & $1(6.7)$ \\
\hline Hypoglobus & $1(6.7)$ \\
\hline
\end{tabular}

Table 1. Patient demographics

EOM, extraocular muscle movement 


\begin{tabular}{|c|c|c|c|c|c|c|c|c|c|c|c|c|c|}
\hline & \multicolumn{6}{|c|}{ Preoperative finding ( $\mathrm{mm}$ ) } & \multicolumn{7}{|c|}{ Postoperative finding ( $\mathrm{mm}$ ) } \\
\hline & ZM & $\mathrm{ZF}$ & 10 & ZS & $\mathrm{ZT}$ & Sum & ZM & ZF & 10 & ZS & ZT & Sum & $P$ value \\
\hline Mean & 7.43 & 1.91 & 4.78 & 4.49 & 0.88 & 19.48 & 0.09 & 0.00 & 0.07 & 1.79 & 0.00 & 1.96 & $<0.001$ \\
\hline \multirow[t]{2}{*}{ Min } & 0 & 0 & 0 & 0 & 0 & 5.1 & 0 & 0 & 0 & 0 & 0 & 0 & \\
\hline & Z1P1 & $\mathrm{Z2P2}$ & Z3P3 & Z4P4 & Z5P5 & DI & Z1P1 & $\mathrm{Z2P2}$ & Z3P3 & Z4P4 & Z5P5 & DI & $P$ value \\
\hline Max & 10.5 & 0.9 & 4.15 & 0 & 0.4 & 10 & 2 & 1.6 & 0.45 & 1.3 & 1.5 & 4.85 & \\
\hline Min & 2 & 7.2 & 0.65 & 2.9 & 3.8 & 24.35 & 0 & 0 & 0 & 0 & 0.2 & 0.6 & \\
\hline
\end{tabular}

\section{Table 2. Preoperative and postoperative measurements for the zygoma}

ZF, Zygomaticofrontal; IO, Inferior orbital rim; ZS, Zygomaticosphenoidal; ZM, Zygomaticomaxillary; ZT, Zygomaticotemporal; SD, standard deviation; Z, point on zygoma surface of $\mathrm{CT}$ image; $\mathrm{P}$, point on surface of zygoma object of presurgical planning; $\mathrm{DI}$, deviation index; Max, maximum; Min,

minimum 


\begin{tabular}{llll}
\hline No $(\mathrm{n}=14)$ & Preoperative Diff. & Postoperative Diff. & $\boldsymbol{P}$ \\
\hline Volume $\left(\mathrm{mm}^{3}\right)^{*+}$ & $3.93(0.35-10.95)$ & $1.05(0.12-3.61)$ & 0.001 \\
Angle of TZ (degree) *+ & & & \\
Anterior $1 / 3$ & $15.94(0.1-89)$ & $5.51(0-30.1)$ & 0.134 \\
Middle & $35.94(0.5-144)$ & $5.74(0.3-21.4)$ & 0.016 \\
Posterior $1 / 3$ & $41.18(5.1-127.3)$ & $5.15(0-18)$ & 0.002 \\
\hline
\end{tabular}

Table 3. Preoperative and postoperative measurements for the orbit

Diff, difference of the affected and the unaffected eye; TZ, transition zone of orbital buttress; *Mean, †Range. 


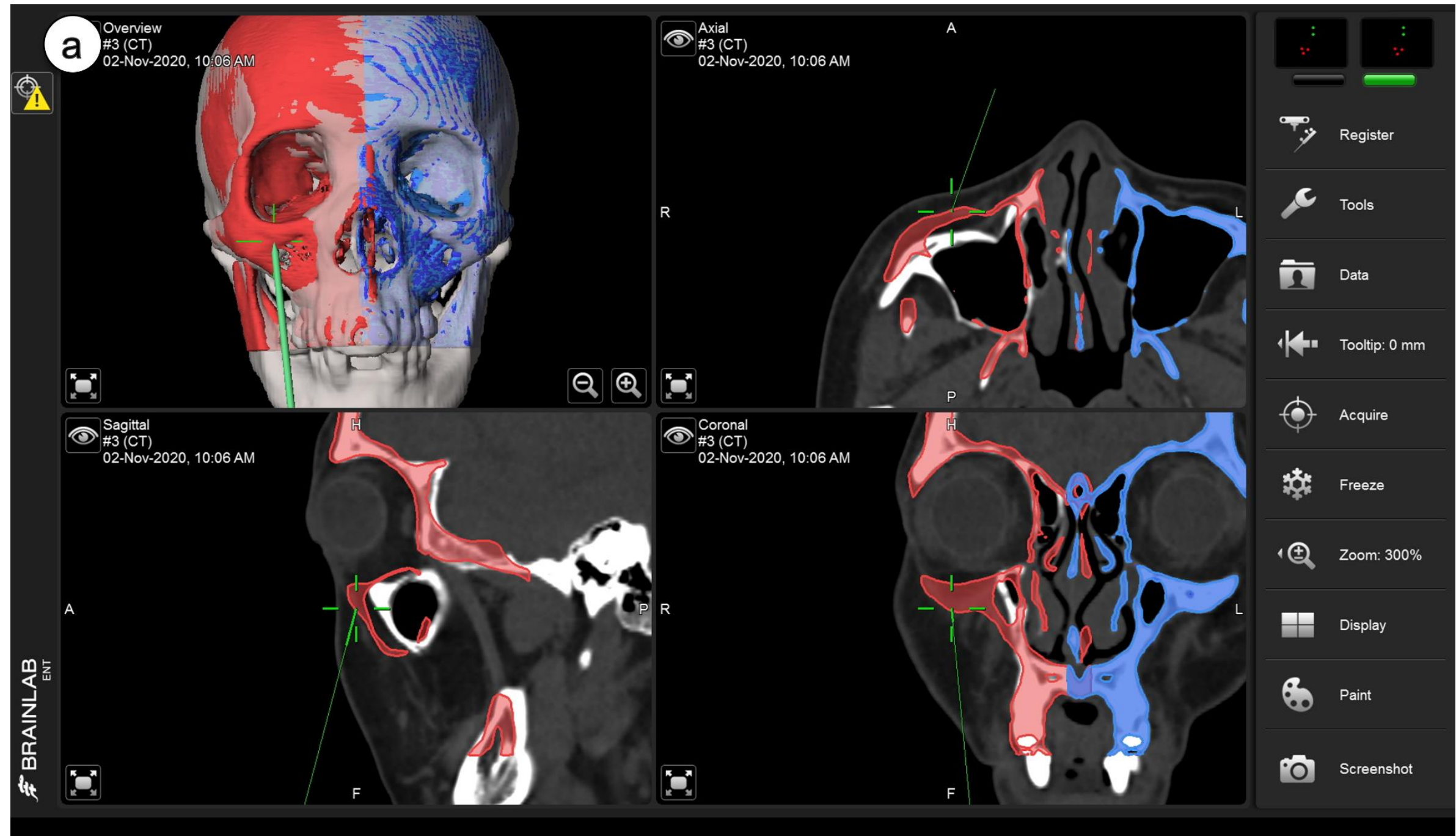


Fig.1b

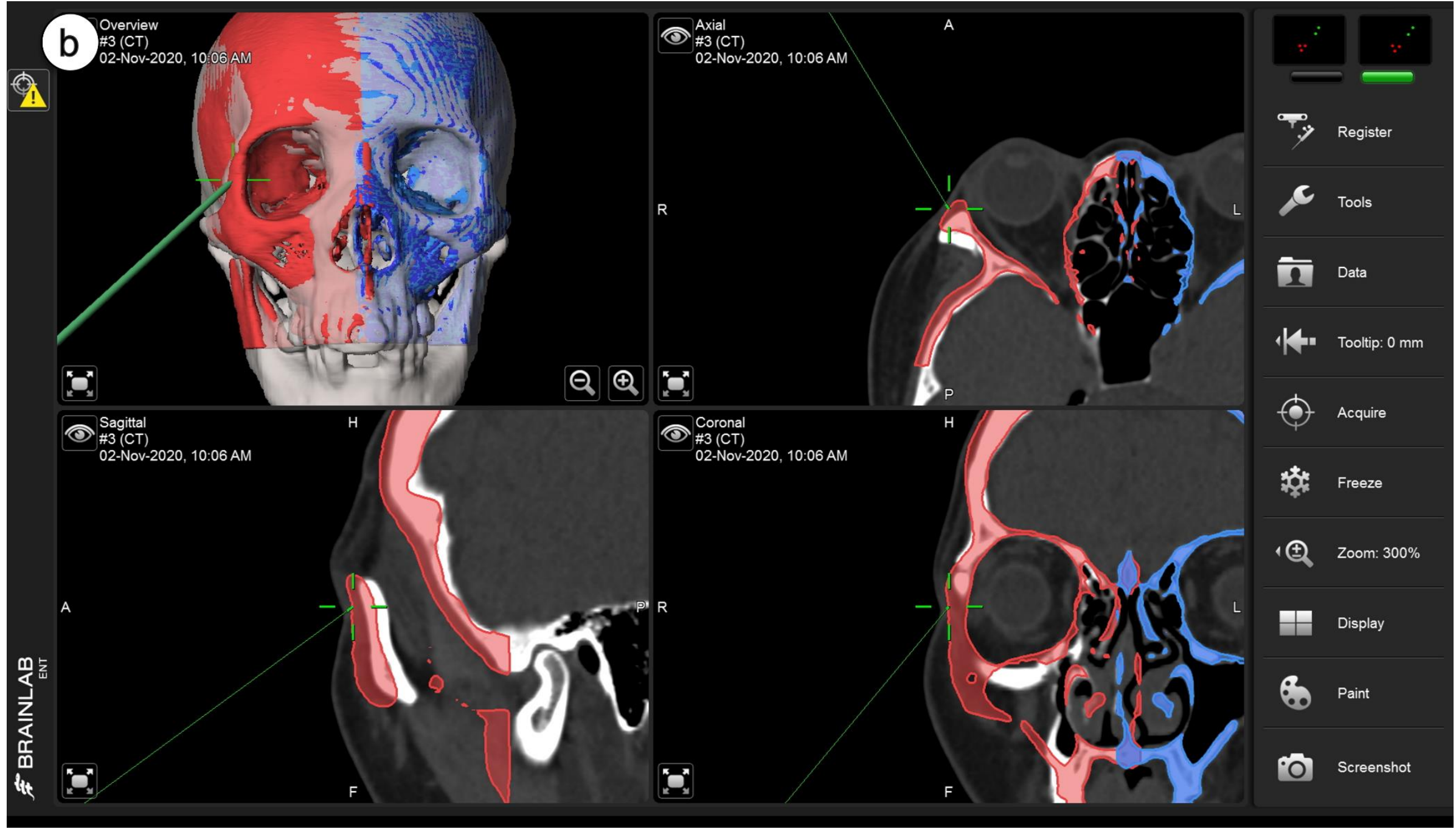


Fig.1c

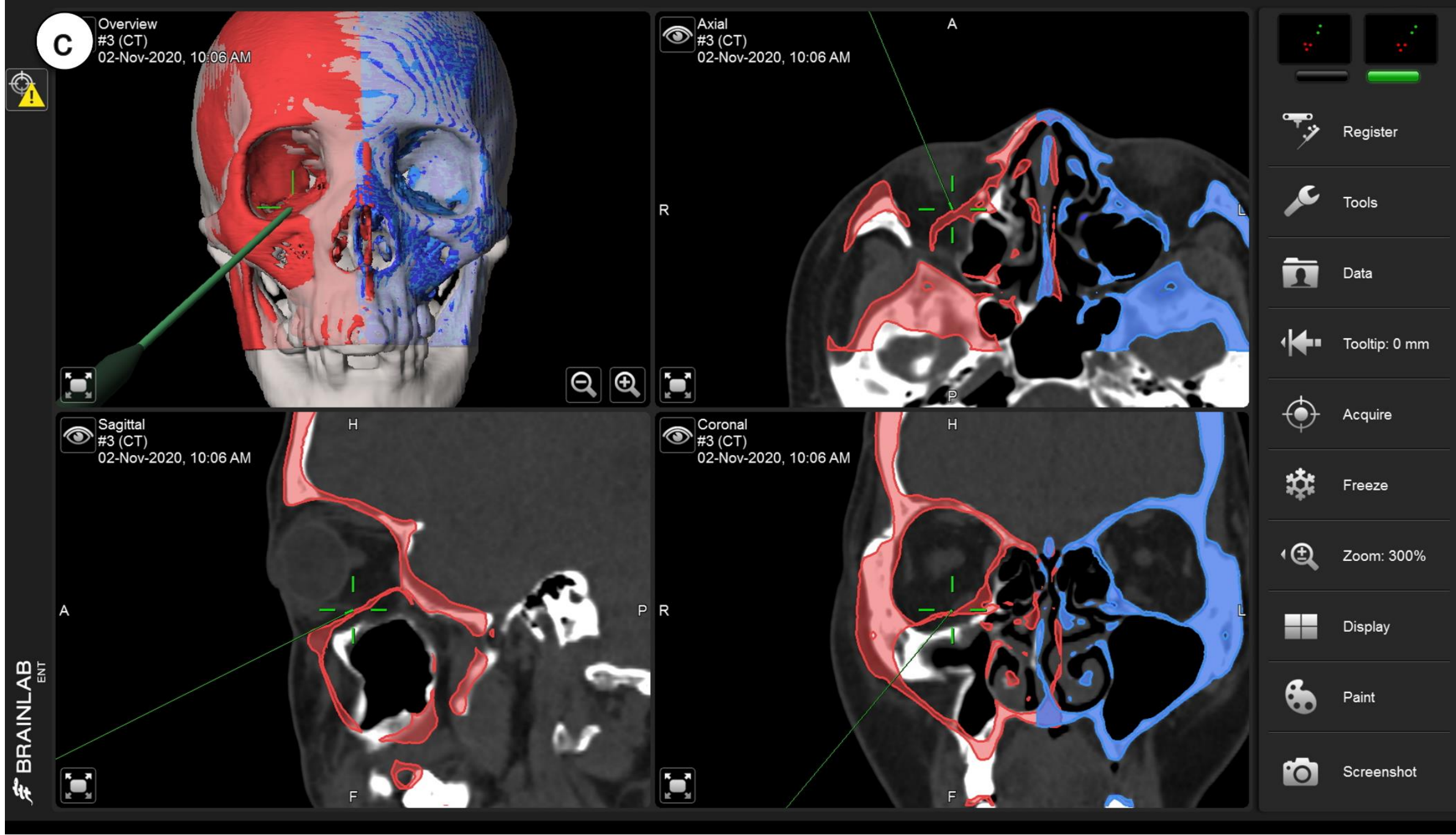


Fig. 2

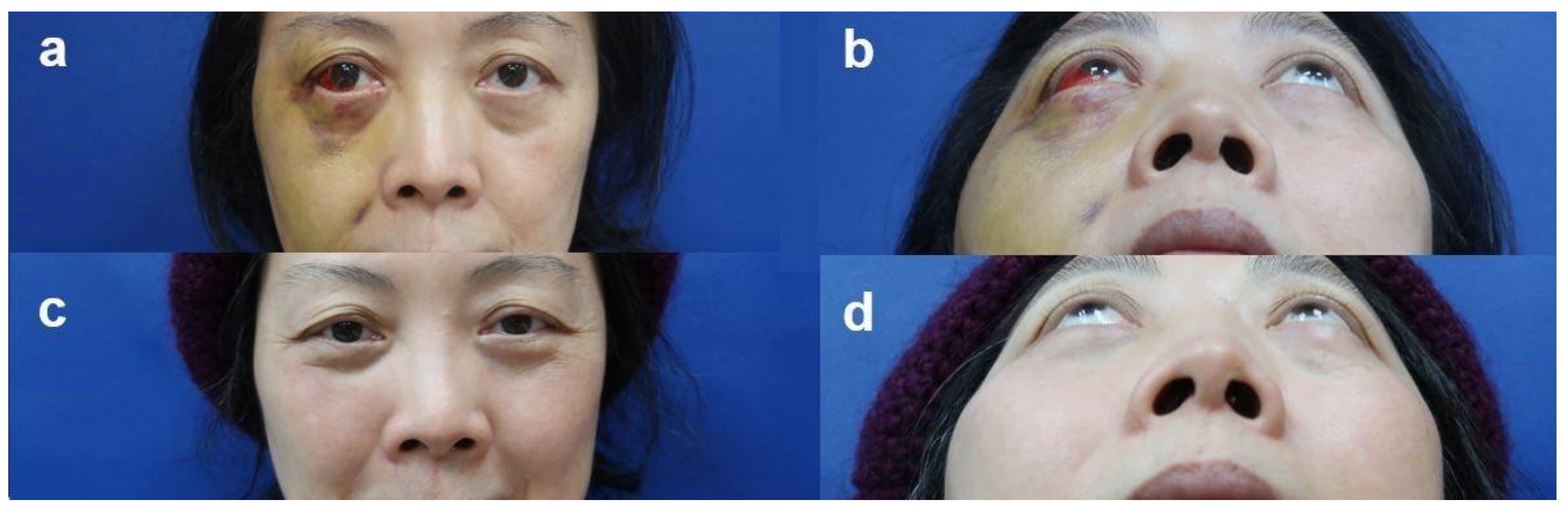




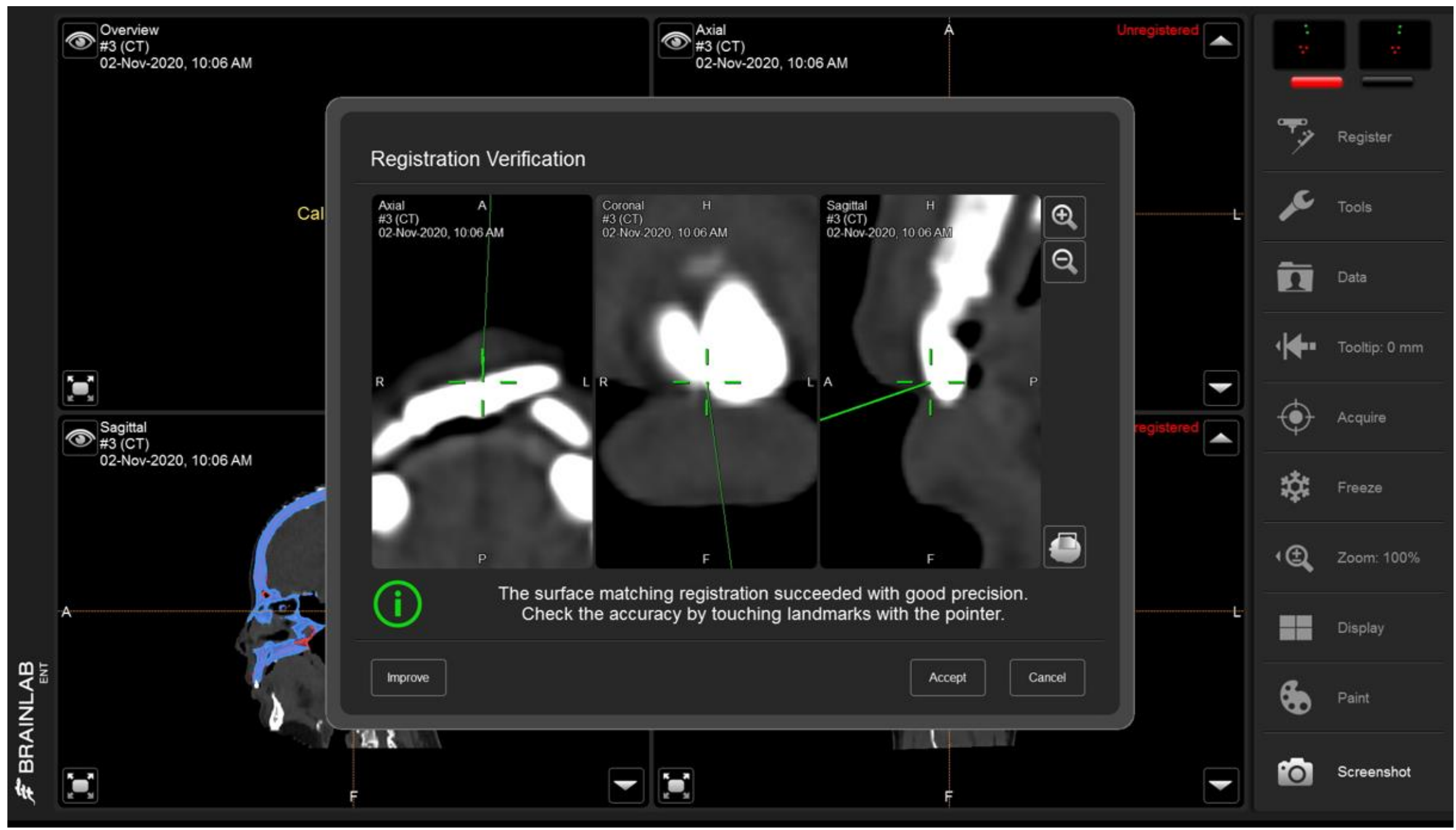


Fig.4 abcd

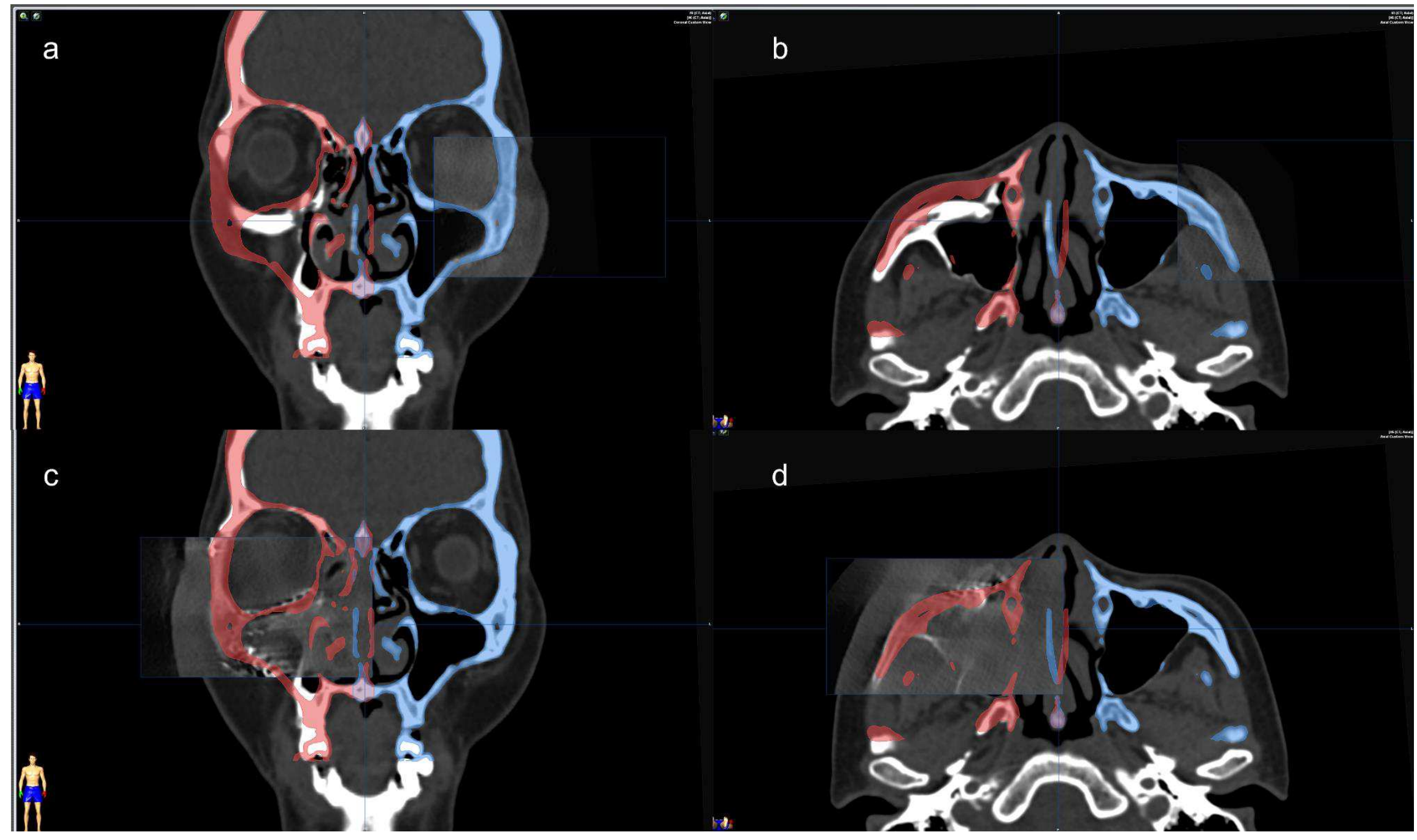


Fig.4 efgh

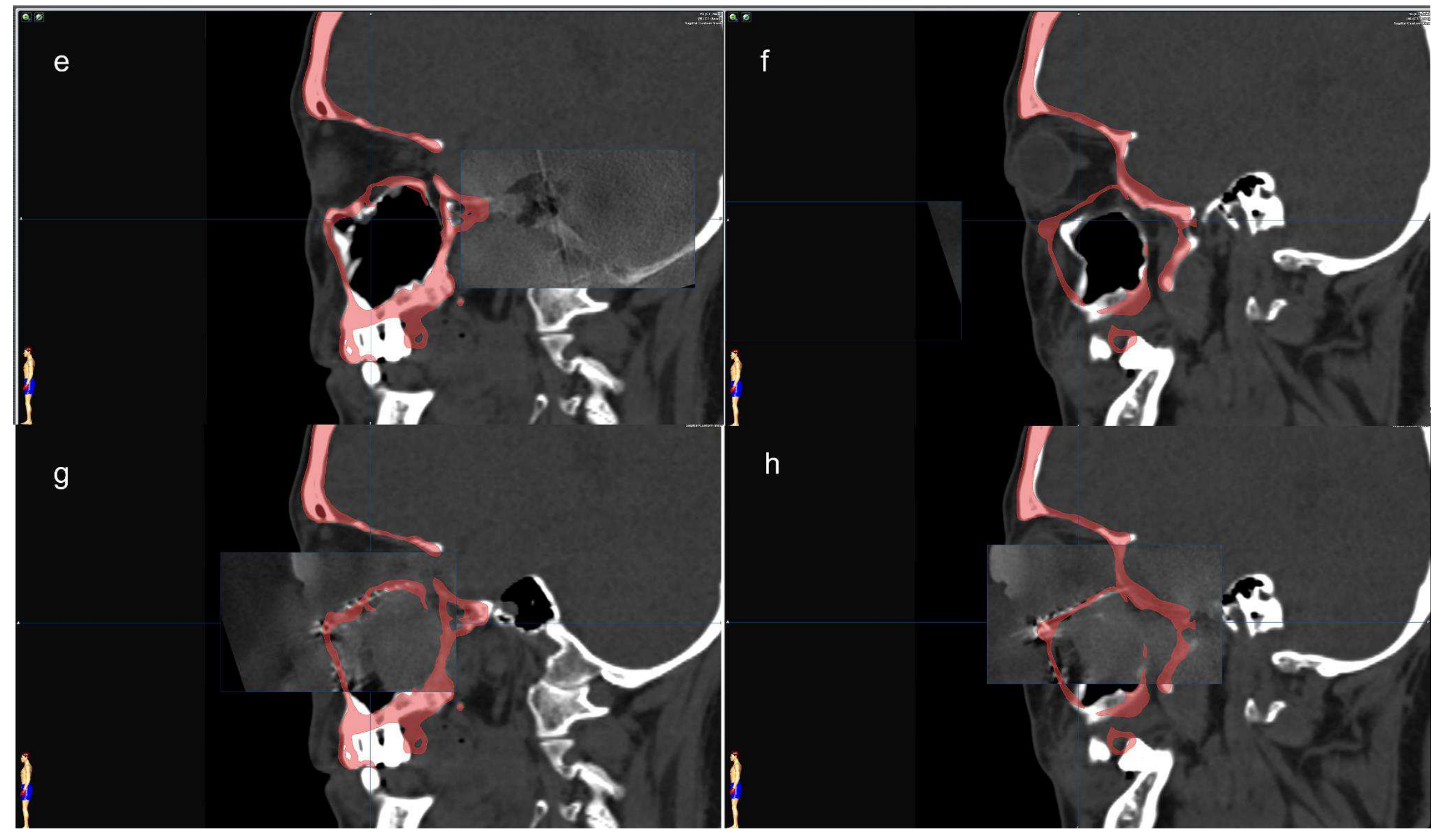




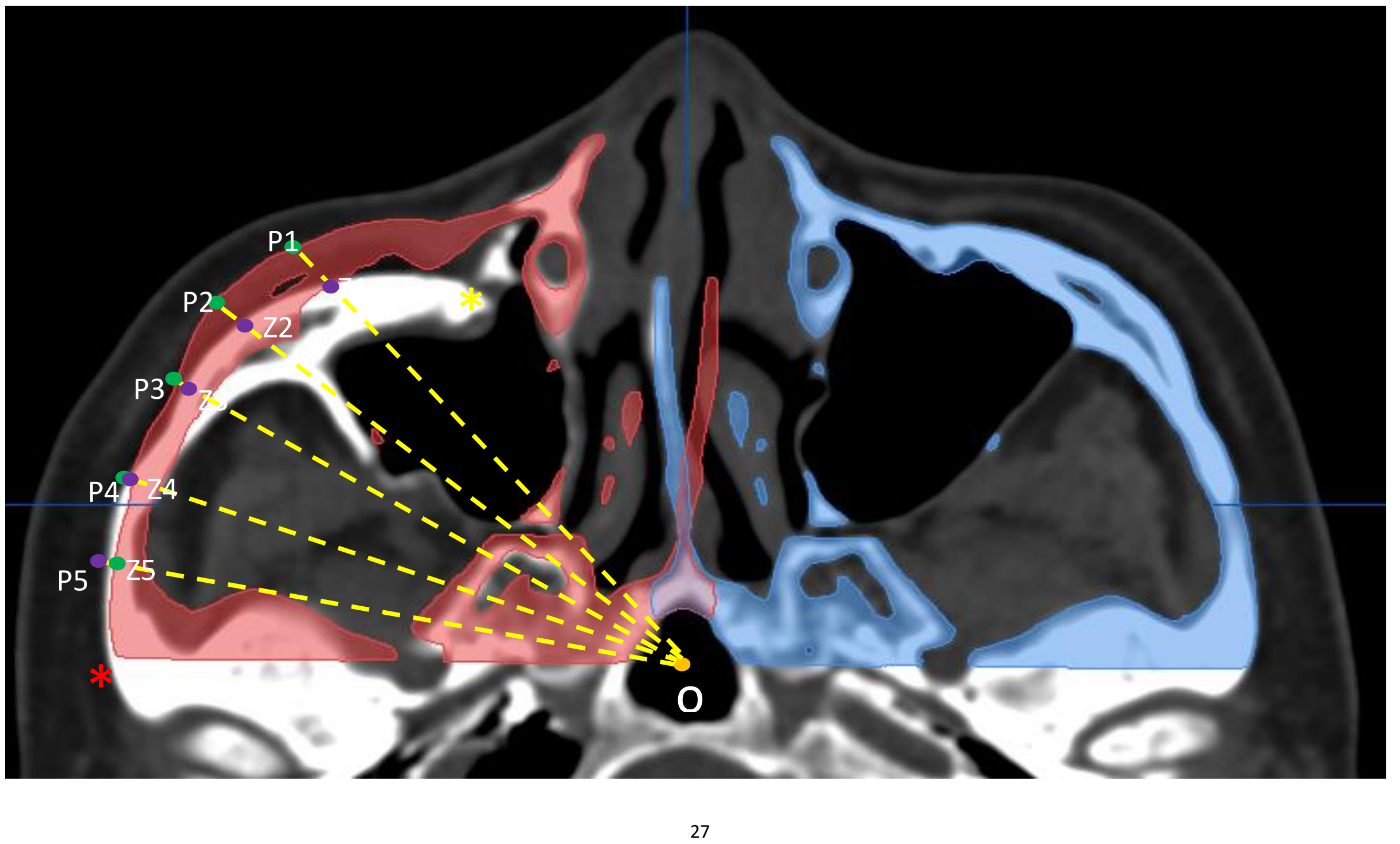




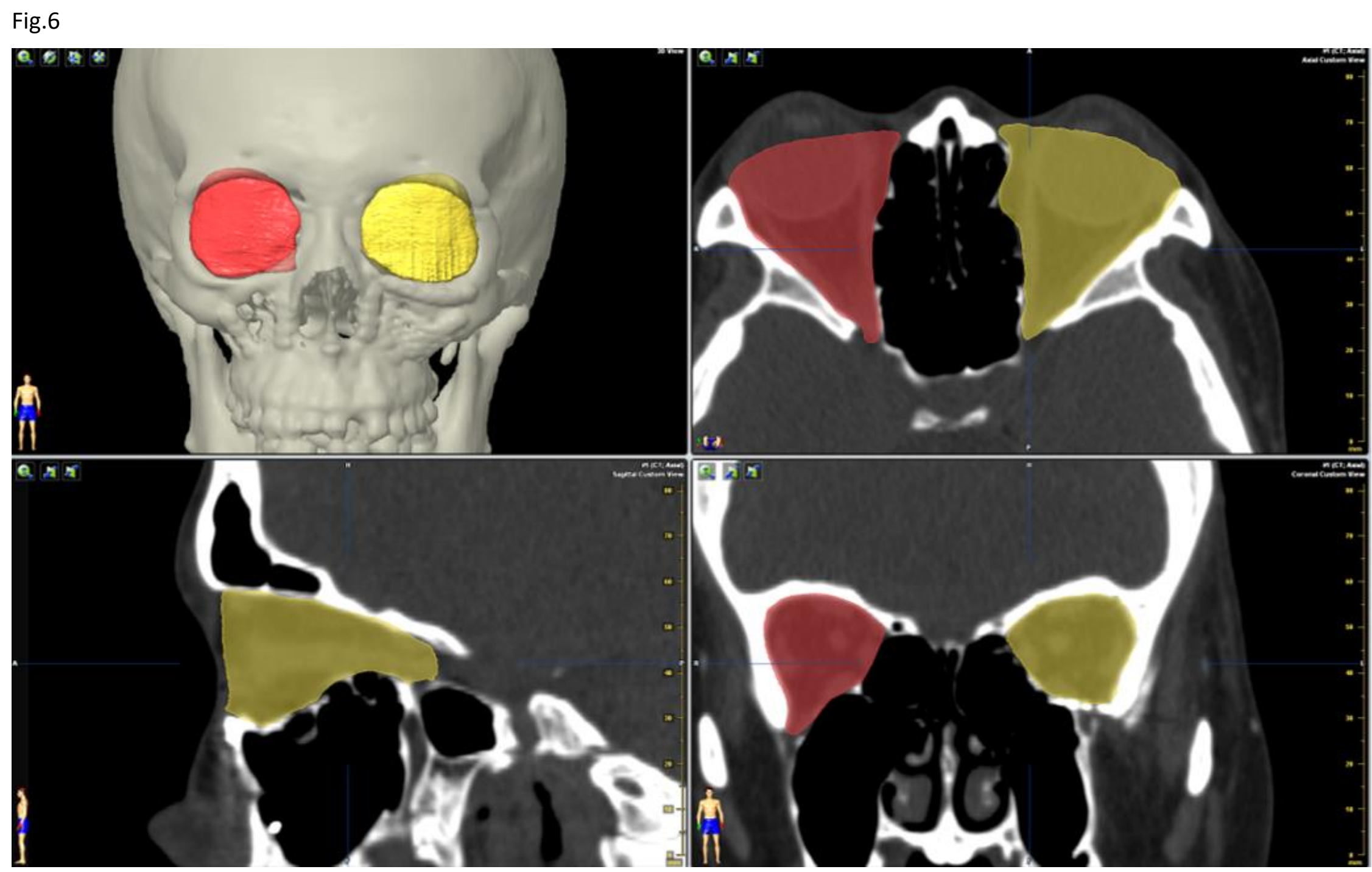


Fig. 7

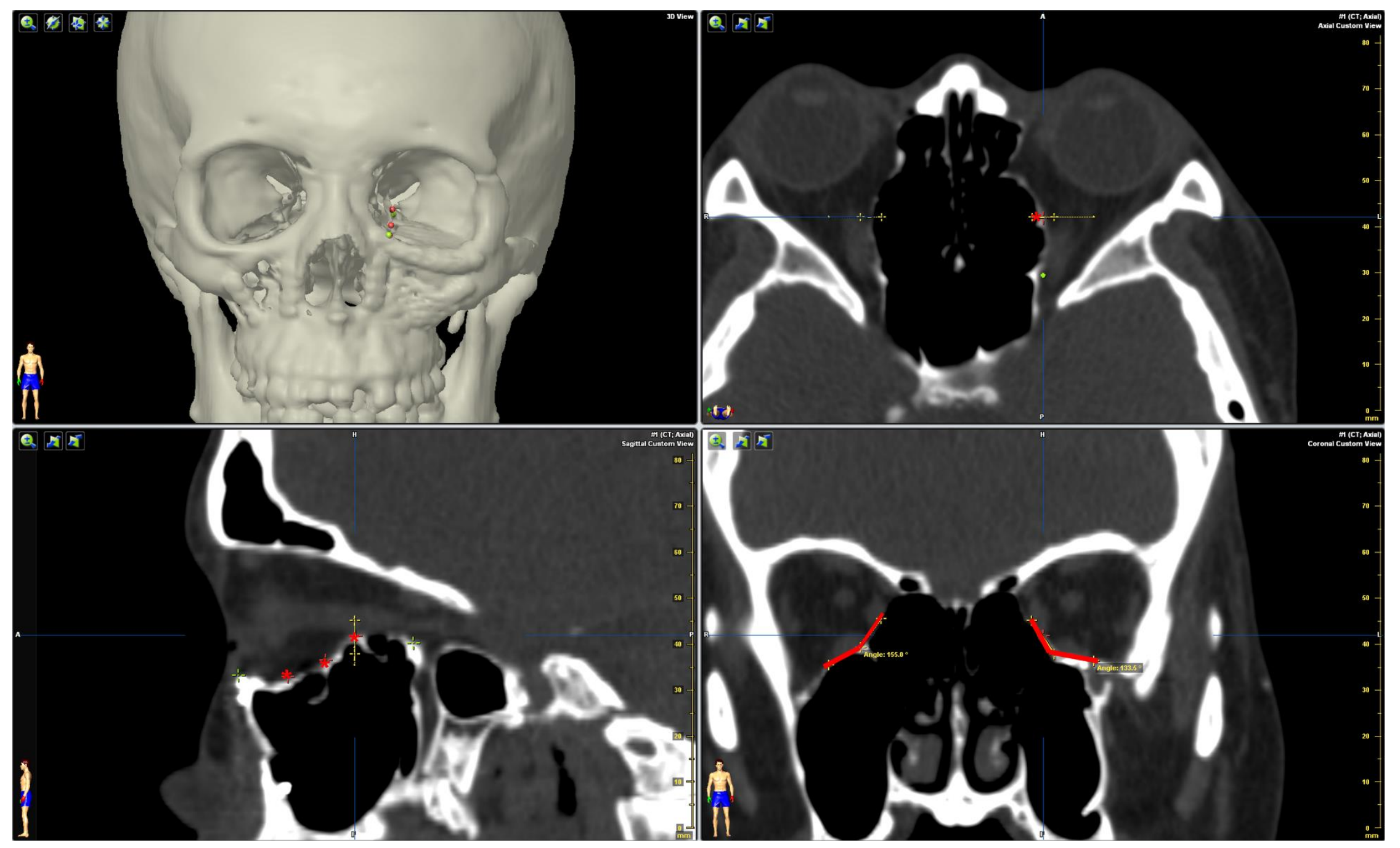

